\title{
Reflexiones en torno a la didáctica de la historia*
}

\section{Reflections on Didactics of History}

\section{Aceneth Perafán Cabrera}

Referencia para citar este artículo: PERAFÁN CABRERA,A. (2013). "Reflexiones en torno a la didáctica de la historia”. En: Revista Guillermo de Ockham 11(2). pp. 149-160.

\section{Resumen}

En este artículo se presentan algunas reflexiones relacionadas con el papel de la didáctica en el proceso de enseńanza-aprendizaje de la historia y de las ciencias sociales y su contribución a la difusión del saber, la comprensión y la explicación de la realidad. Así mismo, se describe el aporte de estas dos disciplinas en el proceso formativo al brindar la oportunidad de cuestionar y reflexionar en torno a los diversos fenómenos del pasado y del presente en sus distintas dimensiones de análisis, favoreciendo con ello el desarrollo de criterios básicos para entender la complejidad que identifica la contemporaneidad. Además, se establecen aspectos relacionados con el avance investigativo que en distintos contextos se ha dado con respecto a la didáctica de la historia.

Palabras clave: didáctica, historia, ciencias sociales, enseńanza.

\section{Abstract}

This article offers some reflections related to the role of the Didactics in the process of teaching-learning of History and Social Sciences and their contribution to the dissemination of knowledge, understanding and explanation of the reality. Likewise, it describes the contribution of history and the Social Sciences in the training process, since they provide an opportunity to question and reflect on the various phenomena of the past and present, in its various dimensions of analysis, favoring the development of basic criteria to understand the great complexity that identifies the contemporary reality. It also establishes aspects of research progress implemented in different contexts related to Didactics.

Keywords: didactics, history, teaching, learning, student.

\section{Introducción}

Esta reflexión es el resultado de un estudio que tuvo como objetivo principal indagar sobre los principales aspectos que identifican el proceso de enseñanza-aprendizaje de la historia en instituciones educativas de Cali, a fin de generar reflexiones en torno a los principales aspectos relacionados con las pautas orientadoras del proceso de enseñanza-aprendizaje: los recursos didácticos utilizados y la incorporación de tecnologías y su relación con la dinámica educativa y los actores presentes en el aula; la participación y motivación

- Fecha de recepción del artículo: 05-08-2013 • Fecha de aceptación: 06-09-2013

ACENETH PERAFÁN CABRERA. Doctora en Sociología de la Universidad de Salamanca. Licenciada en Ciencias Sociales de la Universidad del Valle. Docente del Departamento de Historia, Universidad del Valle, Cali, Colombia. Correo electrónico: acenethperafan@hotmail.com.

\footnotetext{
* Revisión de tema producto de la investigación “Aportes reflexivos en torno a la didáctica de la historia”, adelantado por el grupo de investigación "Asociación Centro de Estudios Regionales -Región-. Facultad de Humanidades de la Universidad del Valle. Colciencias-Categoría A1. Fecha de inicio: marzo de 2011. Fecha de finalización: enero de 2013.
} 
del estudiantado; las concepciones y apreciaciones de los estudiantes respecto de la enseñanza de la historia; y el sentido que atribuyen los maestros a su práctica pedagógica en la enseñanza de la historia y su incidencia en el aprendizaje de los estudiantes.

La investigación se llevó a cabo en cuatro instituciones educativas públicas de Cali, a saber, Santa Librada, Antonio José Camacho, Luis Madina y Álvaro Echeverry Perea, con un total de 150 estudiantes encuestados, matriculados en el noveno grado y siete docentes del área de ciencias sociales.

De esta investigación se derivaron algunos aportes en torno a las buenas prácticas del maestro así como preguntas acerca del desarrollo de la didáctica y su contribución al fortalecimiento del proceso de enseñanza de la historia y de las ciencias sociales. De estos aportes se destacan los siguientes:

- En el campo de las ciencias sociales es necesario formar al estudiantado en conexión estrecha con el contexto, mediante estrategias y contenidos creativos que posibiliten el conocimiento de la sociedad y sus desafíos, logrando de esta forma una verdadera interacción entre la realidad y la escuela.

- Para conseguir lo anterior, es necesario acudir a los aportes que se desarrollen en el campo de la investigación pedagógica, a fin de conocer con mayor profundidad lo que ocurre en las aulas durante el proceso de enseñanza-aprendizaje y así determinar qué significado tienen las ciencias sociales y la historia para el estudiante adolescente de hoy, cómo despertar en el aula el interés de los jóvenes por el conocimiento histórico y de qué forma la historia y las ciencias sociales pueden contribuir a su formación ciudadana.

- El desafío de las ciencias sociales consiste en formar futuros ciudadanos en el marco de un proceso educativo en el que tengan la posibilidad de disponer de información rigurosa, mediante la cual puedan generar reflexiones coherentes y asumir posiciones críticas frente a las diferentes situaciones relacionadas con los retos que la sociedad contemporánea impone y que revisten un enorme grado de complejidad.

Sobre la base de lo anterior se presentan algunas de las conclusiones que se extrajeron de dicha investigación y que hacen referencia al papel de la historia y de las ciencias sociales en el proceso de enseñanza-aprendizaje y a la adquisición, por medio de este proceso, de un pensamiento crítico y contextual particularmente en aquellos estudiantes que logran asimilar los aportes de las ciencias sociales y la historia.

\section{El papel de la historia en la formación}

En los momentos actuales cuando se viven plenamente los efectos de la globalización en el marco de la denominada "sociedad del conocimiento", resulta esencial pensar en las transformaciones que deben efectuarse en el ámbito didácticopedagógico con el fin de involucrar de manera plena al docente en los nuevos requerimientos exigidos hoy en el aula.

Muchos de los problemas que se suscitan en la sociedad del presente hunden sus raíces en difíciles procesos originados en el pasado sobre los que es preciso dar una mirada. Una aproximación rigurosa a la realidad de la sociedad implica adentrarse en el conocimiento de sus principales retos sociales, ambientales, educativos, políticos y económicos y es sabido que en el momento actual son bien conocidos los efectos de la globalización en lo que respecta a la supresión de fronteras y barreras arancelarias y al intercambio masivo de bienes y servicios entre países, lo que ha dado lugar a la creación de un sistema económico mundial que implica la toma de conciencia de las diferencias sociales que este conlleva. Toma de conciencia a partir de la cual se resalten los cambios estructurales que se vienen produciendo en la comunidad humana actual y que no pueden estar circunscritos únicamente al ámbito económico, sino que, por el contrario, deben ir en pos de una mayor justicia social.

Los docentes de historia y ciencias sociales tienen la labor de preparar a los estudiantes a fin de hacerlos más conscientes de la complejidad que han adquirido los actuales fenómenos sociales, económicos, ambientales y políticos como fruto de las lógicas con las que opera la sociedad, y a su vez llevarlos a la comprensión de las diferentes acciones y diversos intereses de los actores que operan en el marco de las dinámicas de análisis.

Los conocimientos aportados por la historia y las ciencias sociales favorecen la consolidación de 
una identidad de individuo, posibilitan su ubicación en el contexto de una realidad temporal y espacial y generan nexos de interacción entre los sujetos en materia de comunicación que posibilitan el reconocimiento, la valoración y el disfrute de aquellos elementos que integran el patrimonio de una sociedad.

Aun cuando estos aspectos permiten reconocer el alcance de la historia en el proceso formativo de los individuos, es necesario resaltar que en algunos contextos educativos los estudiantes no logran vincular los conocimientos adquiridos en historia con aquello que consideran básico para la vida cotidiana; es decir, no hallan la forma de interconectar esos saberes con lo que pasa en su cotidianidad.

\section{La investigación y su papel en las prácticas educativas}

En distintos entornos subsisten en todo el mundo problemas comunes en lo que respecta a la enseñanza de la historia y las ciencias sociales, la mayor parte de los cuales son similares en la manera como se manifiestan, pues tienen causas análogas. Se trata de problemas de índole educativa que muchas veces no son objeto de análisis por parte de los científicos sociales y menos de la mayoría de los ciudadanos. Por tanto, resulta importante indagar acerca de los diversos aspectos involucrados durante el proceso de enseñanzaaprendizaje, pues de esta forma se pueden superar los diversos obstáculos.

$\mathrm{Si}$ bien es cierto que en la actualidad se han dado notables avances en el proceso de enseñanzaaprendizaje de la historia y de las ciencias sociales (principalmente de la historia, pues ya no se exige el relato memorístico que describían fielmente los héroes, fechas y demás detalles de sucesos) no lo es menos el hecho de que en las aulas se toma con poco interés el papel de la investigación como herramienta clave en el proceso de enseńanza de la historia.

Lo ideal es que dentro de los paradigmas de enseñanza de la historia se debe tener en cuenta no solo la formación del docente como historiador y el contexto en el que se educó, sino también a los estudiantes que está modelando a fin de que el proceso educativo brinde los elementos necesarios para la construcción de un conocimiento histórico que faculte tanto al estudiante como al docente para la reflexión y la crítica frente a los procesos sociales estudiados en el aula. En este sentido, es necesario tener presente el papel significativo que cumple el docente en cuanto puede contribuir a que el estudiante adquiera las bases teóricas necesarias que favorezcan una mejor comprensión de la historia.

En la educación básica secundaria resulta esencial velar por que las prácticas pedagógicas implementadas posibiliten que el estudiantado asuma un papel activo, crítico y creativo con respecto a su realidad. Por tal razón, es esencial el óptimo empleo de métodos de enseñanza-aprendizaje que resulten innovadores y vayan más allá del marco tradicional utilizado en el proceso educativo.

En la sociedad actual surgen muchos interrogantes acerca de cómo lograr que el proceso de enseñanza de la historia desarrollado por los docentes, sea el reflejo de un verdadero compromiso con la formación de una nueva sociedad de jóvenes críticos y reflexivos dentro de una dinámica en la que se dé un estímulo efectivo para el logro del aprendizaje.

Las prácticas pedagógicas llevadas a cabo por el docente contribuyen a despertar el interés del estudiantado en los diversos temas, favorecen su reflexión acerca de los procesos que conducen al conocimiento, facilitan su ubicación en el contexto científico-social contemporáneo y su formación crítica, académica y cultural frente a las problemáticas propias de su cotidianidad. Sin embargo, a pesar de la necesidad de investigar las prácticas formativas en historia en la educación básica secundaria son escasos los estudios en ese sentido, en contraste con lo que de manera práctica se lleva a cabo en el aula. Por ese motivo, conocer los sistemas de los maestros se convierte en una necesidad apremiante para descifrar y comprender las concepciones que orientan y el sentido que le atribuyen a la historia en los procesos de enseñanza que implementan en el aula.

\section{Los retos de las ciencias sociales}

La sociedad actual plantea una serie de desafíos frente a los cuales las ciencias sociales no pueden 
permanecer ajenas. Estos están representados en factores tan relevantes como la desigualdad social, las dinámicas propias de la globalización, las problemáticas socioeconómicas, los conflictos ambientales y territoriales y el reconocimiento de las minorías, entre otros, los cuales se convierten en temáticas de primer orden en la enseñanza y aprendizaje de esta área del conocimiento.

El desafío de las ciencias sociales consiste, por tanto, en formar a los futuros ciudadanos en un marco educativo en el que tengan la posibilidad de disponer de información rigurosa que les permita reflexiones coherentes y asumir posiciones críticas frente a las diferentes situaciones relacionadas con los retos que la sociedad contemporánea impone y que revisten un enorme grado de complejidad.

Al corriente de esta lógica, la enseñanza de las ciencias sociales juega un papel relevante al ofrecer propuestas de formación que se desarrollen con base en una fundamentación científica a partir de la cual se atiendan los requerimientos e intereses del estudiantado, así como los problemas centrales de la ciudadanía en general.

Es preciso tener en cuenta el papel de las ciencias sociales en el análisis de la realidad social, pues si se mira desde el punto de vista histórico su función va mucho más de allá de una mera descripción de los fenómenos sociales del pasado y sus aportes brindan la oportunidad de cuestionar y ejercer una crítica frente a ellos dentro de un proceso que reviste elementos centrales como la diversidad, la complejidad y la pluridisciplinariedad y en el que todos los actores sociales cumplen papeles protagónicos.

La enseñanza de las ciencias sociales está en la obligación de activar la inserción crítica del estudiantado y promover actitudes positivas de reflexión, compromiso y comunicación, básicas en la formación ciudadana, para de esta manera superar ampliamente los límites academicistas e instruccionistas.

Muchos de los retos y temáticas de la sociedad contemporánea tienen en las aulas escasa presencialidad. Se ha levantado una barrera entre el aprendizaje académico y aquel que se obtiene a partir de la experiencia proveniente del entorno en el que se desenvuelve el estudiante; por lo tanto, es necesario que la didáctica contribuya a la formación del estudiantado en conexión estrecha con la realidad, mediante estrategias y contenidos creativos que posibiliten el conocimiento de los desafíos de la sociedad y de esta forma crear el nexo entre la realidad y la escuela.

La mirada hacia el pasado resulta importante si se vincula con los retos del presente mediante relaciones con elementos de orden social, económico, ambiental, cultural, político e ideológico. Desde esta perspectiva, los procesos de enseñanza y aprendizaje de las ciencias sociales contribuirán a mejorar la visión que de esta área tiene el estudiantado, al reconocer su funcionalidad para lograr un acercamiento estrecho del conocimiento social en conexión con el entorno en el que se desenvuelve.

\section{La importancia del estudio de la historia}

Un aspecto importante en la dinámica de la enseñanza-aprendizaje de las ciencias sociales y específicamente de la historia, son las características que identifican a las personas a quienes se dirige esta área del saber, dadas las diferencias que se presentan en el contexto socioeconómico, cultural y educativo del estudiante. Como bien establece González (1996) "[...] no es, en efecto, lo mismo enseñar a un alumnado procedente de capas sociales acomodadas, en general bien motivadas y que pueden reconocer en la historia al uso su propia 'historia', que hacerlo a adolescentes procedentes de grupos sociales o étnicos expulsados de la historia tradicional, con la que difícilmente podrán identificarse o encontrarle sentido" (p. 60).

Los estudiantes deben comprender que la verdad histórica se transforma; no es única ni inamovible y está en permanente construcción. Los estudiantes pueden hacer análisis propios en asuntos que sean de su interés.

La enseñanza de las ciencias sociales y de la historia en particular, favorece en el estudiante el aprendizaje de entorno socioeconómico, cultural, geográfico, político, etc., en un marco espaciotemporal a partir del estudio de variables ligadas al aprovechamiento, la producción, la distribución y el uso del territorio, en combinación con categorías temporales. Ello puede alcanzarse mediante el empleo de la investigación como herramienta útil 
y esencial para lograr un verdadero acercamiento a los problemas sociales más sentidos en los contextos analizados por los estudiantes. Así mismo, posibilita el desarrollo de una mirada crítica frente a las interpretaciones sobre la realidad del entorno social, así como el análisis de las acciones de los diferentes actores sociales, sus motivaciones y los procesos que se desencadenan alrededor de cuestiones de orden ambiental, social, cultural, político o ideológico.

La conciencia histórica contribuye a un proceso de transformación social en el que los diversos grupos sociales orientan sus acciones en pro de valores como la justicia, la libertad y la igualdad, en un marco de participación y democracia que lleva a la creación de una identidad y al respeto por los valores culturales legados de un pasado; los cuales resultan valiosos y deben ser defendidos frente al vertiginoso movimiento globalizador característico del momento contemporáneo (Arias y Simarro, 2004).

El conocimiento de la historia, podría decirse, permite recuperar la memoria del pasado y por ende aprender de las experiencias vividas y de las acciones humanas desarrolladas en diferentes ámbitos así como de sus efectos a corto y largo plazo en los distintos grupos sociales. A su vez favorece la capacidad crítica y la reflexión, como lo ilustran Arias y Simarro (2004):

Favorece que el estudiante se ubique dentro de su propio espacio/tiempo, lo mismo que en su contexto social. Contribuye a ampliar el campo de la conciencia personal, lo que repercute en el campo de la conciencia social (por lo que los maestros de la historia deben buscar que los estudiantes se responsabilicen tanto de sus propias acciones como de los hechos sociales).

Ofrece la oportunidad de reflexionar sobre el presente y darse cuenta de que cada uno y todos somos sujetos de la historia y que, a cada momento, la estamos protagonizando y "escribiendo".

Permite darse cuenta de que el presente es el futuro de lo que estudia la historia. Facilita el proceso de la integración personal, por lo que enriquece el inventario de las herramientas que ayudan al estudiante en el camino de la autocreación y, como consecuencia, favorecen la integración social. Habla de la identidad y del sentido de la vida, según cómo se ha ido, se es, y según se es, se será. Puede ayudar a educar para buscar la paz y el respeto de los derechos humanos (p.14).

\section{La didáctica como campo de conocimiento}

El recorrido histórico de la didáctica se ha caracterizado en las últimas décadas por cierto grado de avance y de autonomía respecto de la pedagogía. Como disciplina emergente de la pedagogía, sitúa su objeto de estudio en la práctica pedagógica, aunque intenta posicionarse como ciencia o saber disciplinar aparte. No obstante, este distanciamiento no es del todo absoluto, puesto que procura diálogos continuos con la disciplina pedagógica. En su significación más general, se entiende como "arte de enseñar"; es aprender siendo partícipe de un saber, esto es, aprender haciendo, aprender construyendo.

En sus inicios, enfocaban los esfuerzos educativos en el sujeto-maestro en un contexto donde el educando era un ente pasivo del conocimiento transmitido por el educador. Posteriormente, pensadores pedagógicos modernos (Rousseau, Pestalozzi, Montessori, Dewey) advirtieron la necesidad de acentuar o mudar el interés hacia el educando como actor principal en el proceso educativo y fijar la experiencia sensorial del niño como modo de conocer lo que lo rodea. Se asiste así a un tránsito de la necesidad de enseñar a la necesidad de aprender, evidenciando de esta forma el proceso progresista de la didáctica.

Como una manera de ampliar su horizonte, la didáctica de mediados del siglo pasado indagaba por mecanismos o herramientas que acercaran el conocimiento científico al lenguaje cotidiano del educando a fin de impartirlo en la escuela. Con los aportes de la sicología cognitiva y la sociología, el objeto de estudio se traslada hacia las ciencias humanas "buscando hacer enseñable cualquier discurso". Actualmente, la didáctica ha experimentado un cambio en cuanto a su materia de conocimiento centrada en la educabilidad como un proceso propio del educando en el que el educador busca de alternativas para que el educando halle formas propias de conocer y configurar el conocimiento.

Uno de los principales problemas del campo de acción de la didáctica contemporánea tiene que ver con la incursión de la tecnología en el aula, cuyo fin principal es llevar al educando el conocimiento científico que el maestro no ha podido transmitir. En este marco se produce lo 
que Buitrago (2008,) llama el desvanecimiento o invisibilización del maestro como sujeto político. Esto a su vez lleva implícitas ciertas dificultades que la didáctica busca superar. Entre estas se menciona el acto de comprender cómo organiza el sujeto su pensamiento para con base en ello intervenir en sus procesos de aprendizaje; esto es, avanzar sobre los límites y el potencial del educando. Asimismo, se presentan limitaciones en cuanto a la adecuación de los lenguajes disciplinares, vale decir, hacer que los discursos científicos que han de permear el aula sean más comprensibles para el educando. Otra problemática se refiere al maestro como modelo social que debe seguir el educando en cuanto actor del proceso educativo, ya que es la persona encargada de impartir los saberes culturalmente adquiridos. De igual forma, se busca en el educando un sujeto modelizante en relación al otro que posea un ritmo de aprendizaje diferente (Buitrago, 2008).

Un aspecto que también representa dificultad y se busca superar es el enfoque limitante que adjudica a la naturaleza del maestro rasgos meramente reproductivos o transmisores del conocimiento. En este sentido, se busca otorgar al educador el merecido estatus profesional para lo cual se requiere que el maestro rompa con el paradigma de transmitir un saber y abogue por alternativas formativas que le permitan deslindarse de las lógicas pedagógicas $\mathrm{y}$ culturales con las cuales fue insertado en el entorno educativo, en aras de recuperar el vigor del educando. Se entiende aquí al educador no como transmisor de un saber, sino como agente político frente a la perspectiva de las políticas educativas actuales.

Lo importante dentro del panorama de desarrollo de la didáctica es repensar la realidad y en especial el acto educativo y sus múltiples agentes protagónicos en el aula, como algo cuyo perfeccionamiento jamás concluye; es decir, un movimiento constante que busca trascender los límites y hallar otras respuestas a las distintas realidades de cada actor. En últimas, es un llamado para que la didáctica sea meditada en función de comunidades de sentido que han de converger hacia la unidad en el aula y con ello al acontecimiento históricoconstructivo de una neoestructura sociocultural.

Uno de los aspectos claves que se debe tener en cuenta para dinamizar la enseñanza de la historia, estriba en superar aquella limitada visión que la consideraba como un compendio de datos referidos al pasado que describen los hechos como verdades acabadas y deben ser aprendidos de memoria. En este sentido, resulta indispensable que en el aula se incorpore una perspectiva diferente en el manejo de la historia que permita explicar y comprender no solo cómo ocurrieron los hechos, sino de qué forma se llegó al conocimiento del evento histórico o período del pasado.

La historia no puede ser considerada como un proceso estático, sino como algo que se construye teóricamente día a día a partir del aporte generado por la actividad investigativa cuyo fundamento son los distintos tipos de fuentes primarias: archivos, periódicos, documentos, fotos, monumentos, voces y estadísticas, entre otros.

Prats (1999) considera que desde el punto de vista didáctico la labor de enseñanza se vería favorecida si en este proceso se logra que los estudiantes comprendan los métodos mediante los cuales se construye el conocimiento histórico, referidos específicamente a la recolección de información, la formulación de hipótesis, la clasificación, el análisis, la crítica de fuentes los reconocimientos de las causas y efectos y las explicaciones de los hechos históricos. Con ello se busca fomentar un espíritu crítico y reflexivo que les permita vislumbrar que existen diversas maneras de explicar el pasado y confrontar la realidad.

En lo que respecta a la didáctica, un elemento esencial en el ejercicio educativo es la innovación. Esta debe estar estrechamente ligada al proceso de investigación a fin de generar nuevas visiones que hagan posible el desarrollo de la autorreflexión, la capacidad crítica y la interpretación de distintas prácticas educativas que favorezcan tanto a maestros como a educandos y contribuyan con avances significativos al proceso de enseñanza-aprendizaje.

Los métodos de enseñanza se han transformado notablemente y en razón a ello se han dado pasos importantes en el desarrollo investigativo de profesionales expertos que intentan con sus estudios favorecer el proceso de aprendizaje y destacar el alto nivel de complejidad que reviste el aprender, pues incorpora una actitud reflexiva frente a los conocimientos que cada estudiante adquiere de manera permanente: 
Sin lugar a dudas, hoy coinciden desde diferentes enfoques y perspectivas teóricas, el reconocer la importancia del proceso constructivo de adquisición del conocimiento por parte del sujeto que aprende. La idea de un alumno que podía moldearse y dirigirse fácilmente desde el exterior, ha sido cambiada por la idea de un alumno, que procesa, comprende, interpreta y confiere significados a los estímulos e informaciones que proceden de su entorno (Zamudio et al., 1999, p. 25).

\section{Aprendizaje de los conceptos sociales e históricos}

El propósito principal de las ciencias sociales se centra en proporcionar una fundamentación conceptual y desarrollar aquellas capacidades cognitivas que viabilicen la comprensión de las dinámicas de cada sociedad en un espacio y tiempo determinados e identifiquen aquellos aspectos relevantes en materia social, económica, política, ambiental, ideológica y cultural, así como los legados históricos.

Así mismo, facilita el desarrollo de las habilidades cognitivas que favorecen el conocimiento de la cultura democrática, del respeto y la plena participación en el marco de un entorno educativo en el que el estudiante puede generar actitudes responsables y colaborativas con el medio social y natural. Por otro lado, brinda elementos para que los estudiantes piensen y reflexionen de manera autónoma frente a la realidad en la que se desenvuelven y adquieran bases que les permitan tomar decisiones apoyadas en criterios razonables (Camilloni, 1998).

Dado que los procesos sociales se caracterizan por su alto nivel de complejidad, se hace necesario adentrar al estudiante en la adquisición, el entendimiento y la organización de aquellos conceptos y datos que le permitan alcanzar el nivel de abstracción necesario para comprender la realidad social en su plenitud. En este sentido, Camilloni (1998) establece que el educando precisa desarrollar habilidades en el manejo y aplicación de conceptos y teorías que le permitan:

[...] trabajar con distintos tipos de representaciones mentales; comunicarse, observar, buscar y registrar información; razonar, interpretar y valorar la información en sus aspectos formales y en su contenido temático; trabajar en situación de conflicto con- ceptual y teórico; resolver problemas, y construir opiniones, sostenerlas y, cuando corresponde, modificarlas (p. 185).

Los conceptos históricos son categorías que al ser múltiples y complejas están lejos de ser bien definidas, amén de que hay diversas perspectivas desde las cuales analizar un concepto. ¿Qué hacer, entonces? Frente a ello, una de las posibles vías consiste anclar los nuevos conceptos sobre bases teóricas ya adquiridas por los estudiantes.

En este orden de ideas, las propuestas de enseñanza desarrolladas por cada maestro deben tener en cuenta, en su fase de diseńo y planificación, el papel que juegan los conceptos, vistos como el "[...] conjunto de saberes o formas culturales cuya asimilación y apropiación por los alumnos y alumnas se considera esencial para su desarrollo y socialización" (Coll, Pozo, Sarabia y Valls, 1992, p. 53).

Los conceptos facilitan la comprensión de la realidad por parte de los estudiantes a partir de la información y los saberes propios. En su proceso de evolución cognitiva, el estudiante adquiere cuantiosa información que va organizando en forma de redes conceptuales acerca de lo que vive en su entorno y le confiere sentido al concepto en el momento en que le asigna un significado, cuando ese término puede ser entendido plenamente (Zamudio, Pagés y Benejam, 1999).

La comprensión plena de los conceptos es una de las metas esenciales en el proceso de enseñanza, pues de esta forma la formación proporcionada en el ámbito escolar adquiere sentido y significado y favorece la conexión con la realidad:

Los conceptos históricos presentan características que deben ser tenidas en cuenta, tanto como posible fuente de explicación de las dificultades que los alumnos tienen para su comprensión, como para diseñar estrategias didácticas que faciliten el aprendizaje del alumno. En primer lugar, muchos de los conceptos que se presentan al alumno en la clase de Historia poseen un nivel de abstracción muy elevado. A esto se une la complejidad de muchos de ellos que exige la comprensión de otros conceptos (Carretero, 1995, p. 34).

Las significaciones históricas no son estáticas; por el contrario, se transforman de manera constante y se reformulan de continuo. Por ejemplo, el concepto de democracia ateniense es distinto 
del que se usa en la actualidad ya que encierra un alcance diferente. Para comprender muchos conceptos históricos es necesario conocer y asimilar el contexto en el que surgen o cobran importancia; así, la Revolución Francesa corresponde a un hecho histórico en el que la concepción de igualdad cobra un gran significado y encierra incontables aspectos que se deben analizar en relación con el ámbito en el que surgieron. Cabe resaltar, entonces, el hecho de que el significado de los diversos conceptos históricos hunde sus raíces en el momento histórico, el contexto y la perspectiva historiográfica.

Para Carretero y Pozo (1985), la comprensión de los conceptos históricos representa para los jóvenes adolescentes el tránsito de una visión concreta a una más abstracta y el paso de una perspectiva que los contempla como algo aislado y estático, a una que emplea una visión integradora. Esto apunta a que adolescentes con edades entre quince y dieciséis años conciben el mundo social como una realidad diversa de la que forman parte aspectos sociales, económicos, políticos y culturales, pero desde su enfoque no conciben esa realidad como un conjunto en el que se conectan dichos factores (Carretero y Pozo, 1989).

La incorporación de conceptos históricos en niños puede lograrse a través de metodologías adecuadas que vayan más allá de un trabajo informativo sobre fechas, personajes y acontecimientos. El reto de la enseńanza de la historia implica darles sentido a los argumentos tratados en el aula de clase mediante actividades que engloben la reflexión de los sucesos estudiados, el análisis de los distintos factores que se interconectan en ellos y favorezcan el desarrollo conceptual de los educandos (González, 1996).

En lo que respecta a los contenidos, puede afirmarse que su formulación, organización y secuenciación ameritan un proceso direccionado y el empleo de criterios didácticos, epistemológicos y psicológicos a la hora de pensar en este campo del saber.

Las estructuras de pensamiento construidas por cada estudiante, se encuentran en estrecha interacción con el tipo de información que se maneja en el contexto escolar. Es importante tener en cuenta que el interés o desinterés de los educandos está en correspondencia con el grado de asertividad alcanzado en el proceso de selección y organización de una determinada clase de información. Así mismo, ello podrá facilitar u obstaculizar la relación entre los aprendizajes adquiridos y favorecer o perjudicar la intervención de aquellos sobre la realidad.

Los contenidos seleccionados y su forma de abordarlos durante el proceso de enseñanzaaprendizaje, se constituyen en indicadores que pueden ayudar a identificar el grado de alcance del conocimiento. Por ello, resulta relevante conocer sus avances en cuanto a los aportes generados en el estudiante en materia formativa.

\section{Las nociones de espacio y tiempo en las ciencias sociales}

Es imprescindible lograr el dominio de aspectos básicos a la hora de considerar las actividades didácticas que se van a desarrollar. Entre los elementos fundamentales se destaca el aprendizaje de las nociones de espacio geográfico y tiempo histórico, punto importante para lograr la ubicación en un contexto territorial específico y en un momento histórico con características bien definidas.

La mirada al entorno geográfico posibilita el desarrollo del sentido de pertenencia a partir del conocimiento que se adquiere sobre la realidad contextual a todos los niveles. El estudio de los fenómenos y aspectos geográficos que identifican un determinado contexto, así como de los grupos humanos que lo integran, posibilita el enriquecimiento formativo de los estudiantes, pues favorece el conocimiento de la realidad que identifica el medio físico, en consonancia con las transformaciones resultantes de la interacción entre los procesos humanos y el medio físico.

Uno de los desafíos importantes que debe enfrentar la enseńanza de la geografía corresponde al denominado determinismo geográfico, el cual involucra el empleo de metodologías tradicionalistas centradas en la transcripción literal de fragmentos de textos escolares, la ubicación de sitios geográficos específicos y la localización de accidentes físicos y de las divisiones territoriales, lo que en nada beneficia la comprensión de las dinámicas territoriales y los procesos sociales (Pulgarín, 2011).

Se requiere un cambio en relación con la visión instrumental que se tiene de la geografía, pues el enfoque actual se limita a considerarla únicamente 
como un saber que posibilita la localización espacial mediante empleo de mapas, fotos y demás representaciones, limitando con ello su aporte significativo a la comprensión del estudiante acerca de las dinámicas diversas que se desenvuelven en un espacio geográfico en cuanto al medio social y natural.

La innovación didáctica en la enseńanza de la geografía implica, por tanto, superar las meras representaciones físicas del territorio para dar paso a una geografía en la que sus contenidos prioricen una mirada interpretativa, crítica y reflexiva de las dinámicas socioculturales, políticas, económicas y ambientales que tienen influencia sobre el territorio:

El estudio del territorio como objeto de enseñanza permite visualizar la complejidad del mundo real, pone en evidencia la brecha existente entre los saberes sobre la naturaleza y la sociedad, saberes disociados, parcelados y al ser motivo de enseñanza siguen considerándose igualmente distantes. $\mathrm{Al}$ leer el territorio para comprenderlo, esta condición cambia puesto que en él se encuentran situaciones problema que ameritan estudiarse y desde la escuela convocan a la interdisciplinariedad puesto que los problemas son cada vez más transversales, multidimensionales y pluridisciplinares, en suma globales (Pulgarín, 2011, p. 9).

El estudio del espacio geográfico se traduce, por tanto, en un conocimiento de la realidad enmarcada en un territorio, la cual es transformada de manera continua por las acciones del ser humano. Ello permite ahondar en el conocimiento de las diversas problemáticas resultantes de los procesos de interacción con el medio físico.

En lo que respecta al tiempo histórico podemos decir que resulta ser una noción fundamental en el proceso de aprendizaje y comprensión de la historia:

El tiempo es, en consecuencia, una de las variables esenciales, si no la absolutamente esencial, entre las que integran la definición de la realidad histórica. El proceso que llamamos temporal es el que configura como específica, incomparable con ninguna otra, la existencia humana. Sólo el hombre, como ser autorreflexivo, lleva "dentro de sí" el tiempo. Pero, en realidad, como si fuera una paradoja, el proceso temporal envuelve no sólo lo humano, sino todo lo que existe. El hombre participa del tiempo de la naturaleza, pero hace también del tiempo una "construcción propia". El tiempo es una variable, hemos dicho, o una dimensión, [...] esencial, que configura lo histórico integrada en las realidades sociales. Decimos "integrada" porque no hay realidades sociales sin tiempo (Aróstegui, 2001, p. 209).

El transcurrir del tiempo genera movimiento que ocasiona cambios y resistencias. De ahí la importancia de tener en cuenta los diversos procesos de transformación de los diferentes grupos sociales en sus distintas dinámicas, ritmos y efectos (temporalmente creados), para de esta forma comprender las formas de interrelación de las sociedades con el entorno y reconocer las disímiles maneras de adaptación social, económica, cultural y política desplegadas por las diversas sociedades, todo ello en el marco de una secuencia coordinada en términos espacio-temporales.

Otro elemento significativo consiste en lograr la comprensión espacio-temporal (contextual) de aquellos factores característicos de las diferentes categorías de análisis de un proceso o acontecimiento; por ejemplo, los sucesos a nivel global, regional y local, para de esta forma entender aquellos componentes que en apariencia hacen parte de un acontecimiento aislado e individual englobados en un proceso más general.

La dimensión temporal se constituye en una categoría neurálgica en la disciplina histórica. En ella, el tiempo histórico es concebido como la transformación manifestada en los distintos modos de vida social e institucional a lo largo del tiempo. El ejercicio de abordar la realidad a partir de una dimensión histórica, permite comprender los enlaces existentes entre el pasado, el presente y el futuro, lo que constituye uno de los fines fundamentales de la enseñanza de la historia.

Una sociedad que progresa vertiginosamente, marcada por tendencias mediáticas y tecnológicas y en la que convergen eventos trascendentales o banales, hace difícil para los jóvenes el ubicarse en el marco temporal y entender las lógicas que identifican el pasado y el presente. En este sentido, uno de los problemas más recurrentes entre el alumnado adolescente estriba en su dificultad para lograr la conexión de los actores o personajes históricos con las variables espaciales y temporales que forman parte de los acontecimientos.

Se requieren acciones didácticas que posibiliten el acceso a las diversas nociones y categorías que se enmarcan dentro del tiempo histórico, para lo 
cual se deben enfrentar a dos situaciones: el empleo del tiempo como instrumento de medida (esto es, la cronología) y la comprensión de las categorías temporales.

Se trata de vislumbrar la complejidad que encierra la evolución y duración de los fenómenos sociales cuyos procesos de desarrollo pueden tener similitudes, pero variar en el período de duración en determinados contextos geográficos en razón a factores sociales diversos.

Uno de los conceptos más difíciles de comprender es el de secuencia, el cual corresponde al ordenamiento de los hechos históricos. En el ámbito didáctico, autores como Pluckrose advierten lo útil que resulta el uso de material visual en niños para percibir una secuencia de hechos con cierta espaciación en el tiempo; es decir, que no están cercanos entre sí (Pluckrose, 1993).

En el aula uno de los mecanismos que facilita la comprensión en este sentido, consiste en abordar etapas sucesivas de la vida personal de cada estudiante en el marco de un ejercicio que le permita apreciar y distinguir los cambios ocurridos en determinados periodos, así como resaltar las características propias de cada tiempo.

La adquisición del sentido de los ritmos y de la distinta duración de los fenómenos dentro de una sociedad o diferentes sociedades es, sin duda, uno de los objetivos "finales" de la historia, pero didácticamente no es exigible en todos los momentos y etapas o no al cien por cien, como a veces parece pretenderse (González, 1996, pp. 95-96).

Darles sentido a las fluctuaciones en el tiempo de los fenómenos en la sociedad, es uno de los objetivos centrales de la historia. Cuando se habla de cronología puede afirmarse que:

La cronología, como instrumento de medida, mide pero no explica, permite orientarse en el tiempo, relacionar el antes y el después -la sucesión-, el "a la vez" -la simultaneidad- y permite conocer y situar los cambios de información; es un instrumento para recorrer el tiempo, para circular por él. Es el soporte instrumental del tiempo, pero no el tiempo. Es el cuándo; no el qué (González, 1996, p. 96).

Uno de los factores que genera dificultad en la comprensión de la escala temporal, está relacionado con la división de las grandes etapas de la historia mundial, nacional o regional, puesto que esta fragmentación se basa principalmente en una organización temporal centrada en los hechos político-militares de grandes personajes o de la llamada historia nacional.

Es indudable que el uso de las fechas sirve como referente de los diversos procesos históricos y permite construir un sistema de periodización. Sin embargo, cabe recalcar que el conocimiento histórico no está constreñido específicamente a fechas, sino que se fundamenta en aquellas particularidades que caracterizan un momento histórico y un contexto geográfico específico. La fecha es otro punto de referencia que ayuda a la interpretación de los cambios ocurridos.

Lo ideal en este sentido es que el empleo de las fechas sirva como apoyo a la explicación de la realidad histórica sin caer en el frustrante proceso memorístico. Se trata de introducir al estudiantado en la comprensión del sistema cronológico como fundamento para el desarrollo del conocimiento histórico. Ello es necesario, dado que el tiempo histórico va más allá de un marco lineal cronológico, pues involucra procesos caracterizados por una gran complejidad representados en las diversas variables que en él convergen a nivel social, económico, político, ambiental o cultural.

Visto así, este proceso es muchas veces pasado por alto en las aulas, lo que dificulta la comprensión de las variadas dimensiones que abarca el tiempo histórico y los cambios que ocurren en él. El tiempo histórico es, entonces, un concepto bastante complejo y no es fácil su comprensión por los educandos, a quienes no les resulta factible entender el pasado a partir del presente y analizar unas realidades que hoy les resultan ajenas.

\section{A manera de conclusión}

El aprendizaje de la historia y de las ciencias sociales ayuda a potenciar en el estudiante las capacidades de análisis, reflexión, interpretación, representación, formulación y generalización, entre otras. La didáctica cumple una función central al contribuir al desarrollo de estas habilidades.

En el estudio de los procesos históricos es vital que el alumno comprenda los factores internos y externos que se relacionan de manera directa o indirecta con los actores sociales, así como la 
influencia de los aspectos políticos, económicos, sociales, culturales de una sociedad determinada. Para ello, se debe partir de cuestionamientos claves sobre los que cada educando debe reflexionar, referidos al qué, al por qué, al cómo, al para qué, al quiénes y al cuándo, entre otros.

En su vínculo con el saber histórico, la didáctica adecua el tratamiento de las problemáticas y fomenta la interrelación entre las diversas estructuras de análisis de una sociedad, a saber, económica, cultural, ideológica, social, política, etc. Propicia de igual manera, la explicación causal de los hechos así como la dilucidación intencional de los actores involucrados en cada suceso histórico. Su carácter interdisciplinario viabiliza su interacción con el discurso de otros campos del saber, lo cual resulta útil para el enriquecimiento del tratamiento de las temáticas.
La enseńanza de las ciencias sociales y de la historia involucra un proceso reflexivo por parte del educando en torno a los problemas del ser humano en un tiempo y un espacio específicos. Así mismo, implica un trabajo de motivación al estudiante para que pueda aprender a pensar históricamente y conocer las bases metodológicas con las que se aborda el conocimiento histórico, los valores que representa, su papel en la vida cotidiana y en el ámbito sociocultural, unido a los procesos de reflexión que propicia.

Desafíos como estos tienen que ser afrontados por parte de los profesionales dedicados a la labor educativa a través de todas sus prácticas, en un ejercicio de interacción con el estudiantado mediante el cual se le brinden las herramientas básicas para integrar la investigación en su quehacer formativo.

\section{Bibliografía}

- ANDER-EGG, Ezequiel (2006). Métodos y técnicas de investigación social. Cómo organizar el trabajo de investigación. Buenos Aires: Grupo Editorial Lumen.

- ARIAS Y SIMARRO, Concepción (2004). ¿Cómo enseñar la historia? Técnicas de apoyo para los profesores. México: Instituto Tecnológico y de Estudios Superiores de Occidente (ITESO).

- ARÓSTEGUI, Julio (2001). La investigación histórica: teoría y método. Barcelona: Editorial Crítica.

- BETANCOURT E., Darío (1995). Enseñanza de la historia a tres niveles. Cooperativa Editorial Magisterio. (Colección mesa redonda).

- BONILLA CASTRO, Elsy, y RODRÍGUEZ SHEK, Penélope (1997). Más allá del dilema de los métodos: la investigación en ciencias sociales. Bogotá: Uniandes.

- BRUNNER, Jeronime (1988). Realidad mental y mundos posibles. Los actos de la imaginación que dan sentido a la experiencia. Barcelona: Editorial Gedisa.

- BUITRAGO E., Beatriz Lorena (2008). La didáctica: acontecimiento vivo en el aula. En: Revista Cientifica Guillermo de Ockham 6 (2). pp. 55-67.

- CAMILLONI, Alicia W. de (1998). Sobre la programación de la enseñanza de las ciencias sociales. En: Beatriz Aisenberg y Silvia Alderoqui (compiladoras). Didáctica de las Ciencias Sociales II. Teorías con prácticas. Buenos Aires: Editorial Paidós.

- CARRETERO, Mario (1995). Construir y enseñar las ciencias sociales y la historia. Madrid: Visor.

- CARRETERO, Mario; POZO, Juan Ignacio y ASENSIO, Mikel (1989). La enseñanza de las Ciencias Sociales. Madrid: Visor.

- COLL, César; POZO, José Ignacio; SARABIA, Bernabé y VALLS, Enrics (1992). Los contenidos de la reforma. Madrid: Aula XXI/Santillana.

- EGAN, Kieran (1996). Fantasía e investigación: su poder en la enseñanza. Madrid, MEC, MORATA, 1994. En GONZÁLEZ MUÑOZ, M. C. (1996). La enseñanza de la Historia en el nivel medio. Madrid: Marcial Pons. 
- ESCUDERO MUÑOZ, Juan Manuel (1981). Modelos didácticos. Barcelona: OikosTau.

- FRANZ, Teresinha Sueli (2002). Educación para una comprensión crítica del arte. Un modelo de análisis. En: Revista Arte, Individuo y Sociedad 14. pp. 27-47.

- GONZÁLEZ, Alba Susana (2000). Andamiajes para la enseñanza de la historia. Buenos Aires: Lugar Editorial S.A.

- GONZÁLEZ MUÑOZ, Ma. Carmen (1996). La enseñanza de la historia en el nivel medio. Madrid: Marcial Pons.

- MARTÍNEZ RUIZ, Enrique y MAQUEDA, Consuelo (1989). La historia y las ciencias humanas. Didáctica y técnicas de estudio. Colección Fundamentos 103. Madrid: Ediciones Istmo S.A.

- MORALES MOYA, Antonio (1993). Sobre la historiografía actual. En: Cuadernos de Historia moderna y contemporánea. Madrid, IV.

- NÚÑEZ URIBE, Lida Margarita (2004). Reflexiones sobre la enseñanza de la historia desde una experiencia docente en el Instituto Pedagógico Arturo Ramírez Montúfar IPARM- 1. En: RODRÍGUEZ, J. G. Rutas pedagógicas de la historia en la educación básica de Bogotá. Bogotá: Universidad Nacional de Colombia. Programa RED e Instituto de Investigación Educativa y Desarrollo Pedagógico (IDEP).

- PEÑA, Brhiter (2004). La enseñanza de las Ciencias Sociales. En: RODRÍGUEZ, J. G. Rutas pedagógicas de la historia en la educación básica de Bogotá. Bogotá: Universidad Nacional de Colombia. Programa RED e Instituto de Investigación Educativa y Desarrollo Pedagógico (IDEP).

- PORLÁN, Rafael (1993). Constructivismo y escuela. Hacia un modelo de enseñanza-aprendizaje basado en la investigación. Sevilla: Díada.

- PLUCKROSE, Henry (1993). Enseñanza y aprendizaje de la historia. Madrid: Morata/MEC.

- PRATS CUEVAS, J. (1997). La selección de contenidos históricos para la educación secundaria. Coherencia y autonomía respecto a los avances de la ciencia histórica. En: IBER: Didáctica de las ciencias sociales, geografia e historia IV (12). pp. 7-18.

- TOPOLSKY, Jeretz (2003). La estructura de las narrativas históricas y la enseñanza de la historia. En: CARRETERO, M. y F. VOSS, J. Aprender y pensar la historia. Buenos Aires, Amorrortu.

- TOVAR ZAMBRANO, Bernardo (2004). La historia en el aula. En RODRÍGUEZ, J. G. Rutas pedagógicas de la historia en la educación básica de Bogotá. Bogotá: Universidad Nacional de Colombia. Programa RED e Instituto de Investigación Educativa y Desarrollo Pedagógico (IDEP).

- ZAMUDIO, José Ignacio; PAGÉS, Joan y BENEJAM, Pilar (1999). La enseñanza de las ciencias sociales, historia y geografia. Santiago de Cali: Universidad Santiago de Cali.

\section{Webgrafía}

- GARCÍA PÉREZ, Francisco Florentino (15 de mayo de 2000). Un modelo didáctico alternativo para transformar la educación: el modelo de investigación en la escuela. Recuperado en enero de 2013 desde http://www. ub.edu/geocrit/sn-64.htm.

- PRATS CUEVAS, Joaquín (21 de junio de 2007). La historia es cada vez más necesaria para formar personas con criterio. Recuperado en octubre de 2013 desde: http://www.ub.edu/histodidactica/images/documentos/ pdf/historia_necesaria_formar_personas_criterio.pdf, 2007.

- PULGARÍN SILVA, Raquel (2011). Los estudios del territorio y su intencionalidad pedagógica. Recuperado en octubre de 2013 desde: http://www.revistas.una.ac.cr/index.php/geografica/article/view/2798, 2011. 